\title{
The validation of automatic methods for measuring conversational speech activity
}

\author{
P. D. BRICKER and S. PRUZANSKY \\ Bell Laboratories, Murray Hill, New Jersey 07974
}

\begin{abstract}
Sophisticated electronic systems have been developed to measure speech activity patterns automatically, but their accuracy is unknown. The purpose of the present work is to evaluate the fidelity with which a class of computerized systems matches the measurements made by a human observer. With all parameters optimized, it was found that: (1) about $98 \%$ of samples (sampling rate $=200 / \mathrm{sec}$ ) were classified the same way by the system and the criterion method; (2) distributions of utterance durations and speaker-switch intervals were accurately rendered by the automatic system; (3) average durations of talkspurts-bursts of speech activity within utterances-were closely approximated, but the system tended to overestimate the number of brief ( $\leqslant 100 \mathrm{msec}$ ) talkspurts; (4) the system was subject to considerable qualitative error in the measurement of within-utterance pauses and of simultaneous talking.
\end{abstract}

Ordinary conversation provides a rich vein of material for study by scientists of a variety of disciplines. Many of the more abstract aspects of dialogue, such as Wish's (1979) dimensions of dyadic interaction and Bales' (1950) categories, require a high degree of inference on the part of observers and experimenters for their specification and measurement. Other phenomena, such as the literal verbal content and linguistic and paralinguistic categories, are of a more explicit nature. Among the most concrete of these is the temporal pattern of speech and silence. It would seem a straightforward matter to determine, at any moment during an episode of dyadic interaction, whether or not each participant was speaking-so simple, in the view of many investigators, that they have attempted to relegate the task to a machine. The question of how well machines perform this task is the subject of this paper.

Two techniques have been used to obtain the basic measurements of the on-off pattern of speech. The visual techniques (e.g., Goldman-Eisler, 1956; Norwine \& Murphy, 1938; Höhne, Note 1) first convert the speech signal to a visible record, such as an oscillogram, sound spectrogram, or sound motion picture. An observer then reads the beginnings and ends of speech events from these records, which ideally include time reference marks. The automatic acoustic techniques (Brady, 1965; Cappella \& Streibel, 1979; Cassotta, Feldstein, \& Jaffe, 1964; Phillips, Treurniet, Tigges, \& Lewis, Note 2) work with the electrical form of the speech signal, processing it in real time and making an on-off decision at each successive sampling interval.

Visual techniques are inherently capable of yielding very accurate measurements, but are exceedingly timeconsuming. Automatic acoustic techniques, on the other hand, can produce data virtually in real time, but these techniques are subject to the errors inherent in any process that discriminates between signal and noise, as well as errors due to some peculiar characteristics of the speech signal. The accuracy of automatic methods is, in fact, unknown.

The primary purpose of the present investigation is to estimate the error for a class of automatic techniques by comparing automated (acoustic) and manual (visual) measurements of the same samples of conversational speech. Data are presented on how accuracy is affected by variation of the parameters of the automatic system.

\section{METHOD}

\section{The Conversations}

Subjects. Six adult subjects participated in three conversations scored in this study. Subjects A and B (males) conversed for $18.4 \mathrm{~min}$. The topic was Subject B's thesis research. Subjects $C$ (male) and D (female) conversed for $15.4 \mathrm{~min}$ on general topics, especially the contrast of customs between countries. Subjects E (male) and F (female) conversed for $11.3 \mathrm{~min}$ on general topics, with an emphasis on their individual future plans.

Setting. Subjects seated in separate sound booths in adjoining rooms communicated by means of a standard telephone line. Recordings of each subject's voice were made on separate channels of a two-channel Ampex PR 10-2 at $7 \frac{1}{2} \mathrm{in} . / \mathrm{sec}$. The voice signals were picked up by a high-quality dynamic microphone mounted about 18 in. from the subject's lips.

\section{Definitions of Basic States}

The first objective of any speech-activity scoring scheme is to divide the period of time during which a converser is observed into alternating intervals of activity and inactivity. Active periods are defined as "talkspurts" and the inactive periods are "pauses."

Included in talkspurts are periods of fluent speech and other vocal sounds, such as laughter, floor holding hesitation sounds (filled pauses), back-channel signals (Duncan, 1974), and nonverbal expressions of evaluation, surprise, and so forth. Included in pauses are hesitation pauses, speaker-switching pauses, and periods of listening. The intent is to exclude from "pauses" only those silences due to stop consonants.

\section{Scoring}

Criterion method. The first step in the scoring process was 
the creation of typed transcripts of each of the conversations. Oscillograms were then made from the recordings on a Honeywell Visicorder Model $906 \mathrm{C}$ at a chart speed of $5 \mathrm{in} . / \mathrm{sec}(12.7 \mathrm{~cm} / \mathrm{sec})$ and a bandwidth of $4 \mathrm{kHz}$.

Our observer, a female college student, was given brief training in the interpretation of oscillograms. She was then assigned the task of correcting the typed transcripts and transcribing them directly onto the oscillograms. The observer listened to the audio tape recording as an essential part of the performance of this task and then made a second pass through the oscillograms to measure the intervals. She again listened to the audio tape playback, this time as the fundamental criterion for determining the boundaries between talkspurts and pauses. All such boundaries were marked with vertical lines on the oscillogram and the intervals measured using a special ruler calibrated in $5-\mathrm{msec}$ increments. The resulting ordered series of interval durations was then entered into the computer, thus creating a digital record of each subject's times of transition between active and inactive states. For later computational and comparison purposes, each of the storage-efficient records was converted to a binary time series with a sampling period of $5 \mathrm{msec}$. That is, each active interval was converted to a string of "ones" equal in length to its duration in (5-msec) units, and each inactive interval was similarly converted to a string of "zeros." Strings of zeros alternated with strings of ones in this representation, and the sampling intervals were serially numbered from a point designated as the beginning of the conversation. This starting point was the same for both participants. These time series, referred to as "criterion data files," contained a complete record of each subject's talkspurts (strings of ones), pauses (strings of zeros), and absolute times of transition between states.

We did not follow the usual practice of using a second observer to obtain the measure of the reliability of the scoring method. However, our observer consulted with the experimenters on sequences that were difficult to classify. Furthermore, every discrepancy between the criterion method and the better automatic methods was reevaluated by the observer and, when necessary, by one or both experimenters. The criterion scoring then represents, in effect, agreement by a committee of three.

Automatic scoring system. Figure 1 shows a block diagram of the automatic scoring system. The output of each speech source was first rectified and smoothed with a very short $(5-\mathrm{msec})$ time constant and then detected. The dual detector (Jensen, Note 3) was a variable-threshold device that converted all suprathreshold signals of each speaker to one voltage level and all subthreshold signals to another; summing the two detector voltages produced four discrete levels at the output. The detector output was then sampled by an analog-to-digital converter at a $200-\mathrm{Hz}$ rate to form a raw data file with the same resolution as the observer's $(5 \mathrm{msec})$. The four discrete levels in the raw data file may be unambiguously interpreted in terms of whether each subject was speaking (State 1) or silent (State 0) at each sample point. A computer program then converted each raw data file into two time series, one for each subject in the dyad. The same

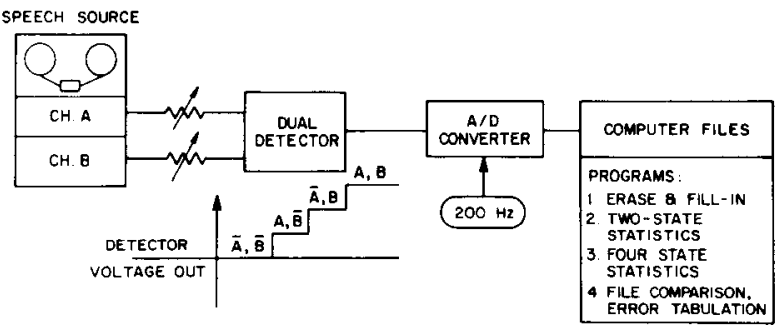

Figure 1. Block diagram of automatic speech-activity scoring system. program also performed digital smoothing operations on each time series. The smoothing operations were governed by three parameters:

Initial fill $(I F)$. Strings of zeros in the raw data file equal to or less than that value in length were replaced with ones; this operation corrected for gaps caused by below-threshold samples during the glottal cycle of low-pitched voices.

Erase (E). Strings of ones equal to or shorter than the value chosen for this parameter were converted to zeros; the goal was to erase isolated bursts of noise, either electrical or acoustic but not vocal.

Final fill $(F F)$. Strings of zeros equal to or less than this value, remaining after the IF and $E$ operations, were converted to ones. The objective was to bridge gaps caused by stop consonants.

The three smoothing parameters, temporal or E-F parameters, are realized as a part of the software. The fourth parameter was the detector threshold. The detector threshold was part of the hardware; therefore, a separate raw data file had to be made for each value of the threshold parameter. For each of four values of detector threshold $(-28,-24,-20$, and $-16 \mathrm{dBm})$, the three conversations were processed with either 0 or $10 \mathrm{msec}$ initial fill, 15 or $30 \mathrm{msec}$ erase, and values of final fill ranging from 150 to $350 \mathrm{msec}$. A subset of five combinations of E-F parameters has been selected for illustrative purposes. That is, the recording of each subject's side of the conversation was converted into 20 different time series ( 4 thresholds by 5 sets of E-F parameters), each of which had the same form as the criterion data file and was referred to the same starting point and time base. We shall refer to these machine-scored time series as "MS data files." Again, the actual data storage was compact, but the time-series form, as will become clear, was used to compare each MS data file with the criterion.

Note that the present technique and four others (Brady, 1965; Ramsay \& Law, 1966; Höhne, Note 1; Phillips et al. Note 2) differ from conventional mechanized acoustic methods in that the bulk of the smoothing is done digitally, rather than by means of a large time constant before the detector. Analog smoothing leads to the artificial lengthening of talkspurts. Digital smoothing avoids this problem because the FF operation ends a string of ones where it ended on the raw data file, if no more ones occur within a number of samples equal to the current value of the FF parameter.

\section{Comparison Procedures}

The evaluation of any given set of parameter values for automatic scoring amounts to comparing two time series: namely, the criterion data files and the MS (machine-scored) files. The basic comparison technique involved aligning the MS time series under test with the corresponding criterion time series and combining them point by point to create another time series, called the "difference series." Each element of the series contained two bits of information at each sample point; both the relationship between the criterion and test series (agreement or disagreement) and the state of the criterion (active or inactive) were encoded. The pattern of criterion states before, during, and after each string of disagreements uniquely classified each error as to whether it was too long or too short (for quantitative errors), an extra or missing event (for qualitative errors), and talkspurt or pause (for extra or missing events). The computer program measured the length of each error run in the difference series and stored the value in one of eight arrays as determined by the pattern of criterion activity values. The computer program prepared various summaries of the error statistics and also output the difference series for later analysis of the effects of qualitative error on the statistics of the original talkspurts and pauses.

In what follows, we rely chiefly on counts of qualitative errors, expressed as proportions of the relevant number of events in the criterion file, to evaluate the classification performance of 
a set of parameters. Quantitative error is assessed by examining the effects of changes in parameters on empirical cumulative distribution functions (ECDFs) of talkspurts and pauses. At this stage, we are concerned primarily with the ability of mechanized techniques to render the on-off patterns of individual speakers; in the section on dyadic phenomena we will turn to questions of more interest to students of social behavior, when we examine measurements of interactive events derived from these basic patterns.

\section{TWO-STATE RESULTS}

Results for each of the six talkers are examined on three levels: (1) the sample-by-sample level, (2) the event (or qualitative) level, and (3) the quantitative level. At the sample-by-sample level, comparison is straightforward. An automatic scoring either agrees or disagrees with the criterion at each sample point. The performance measure, proportion of samples in error, is simply the number of ones in the difference series divided by the number of samples. At the event or qualitative level, comparison is more complicated, but errors are still unequivocally defined. For general summarization, we used the ratio of the total number of qualitative errors to the number of talkspurts in the criterion series (QE/TS). We also examined the effects of parameter changes on the number of extra and missing talkspurts and pauses and on the total number of events. At the quantitative level, empirical cumulative distribution functions (ECDFs) of candidate and criterion scorings were compared in terms of their location and shape.

\section{Sample by Sample}

Figure 2 shows total proportion of samples in error as a function of threshold, with erase and fill values as parameters, for each of two talkers (C and E). These are typical cases, with talkers varying chiefly in how steeply their error functions rose at the highest and lowest thresholds and in whether $-24 \mathrm{dBm}$ or $-20 \mathrm{dBm}$ was a more appropriate threshold. These data suggest that final fill values of $200-250 \mathrm{msec}$ and thresholds of -20 to $-24 \mathrm{dBm}$ are to be preferred over the more extreme values. As a practical matter, it appears that very good performance-about $98 \%$ of samples classified correctly-can be achieved by a properly adjusted device of the type under study.

\section{Qualitative}

Figure 3 shows plots of the ratios QE/TS for the same two speakers as those in Figure 2 as functions of thresh. old, with fill-in and erase values as parameters. Both of these cases illustrate two points. (1) Threshold and final fill interact in the expected manner. That is, more final fill improves the performance of a threshold set too high, whereas less final fill compensates to some extent for a too sensitive detector. (2) In general, final fill values in the range of 200-250 msec perform better than larger or smaller values. The two cases are chosen to

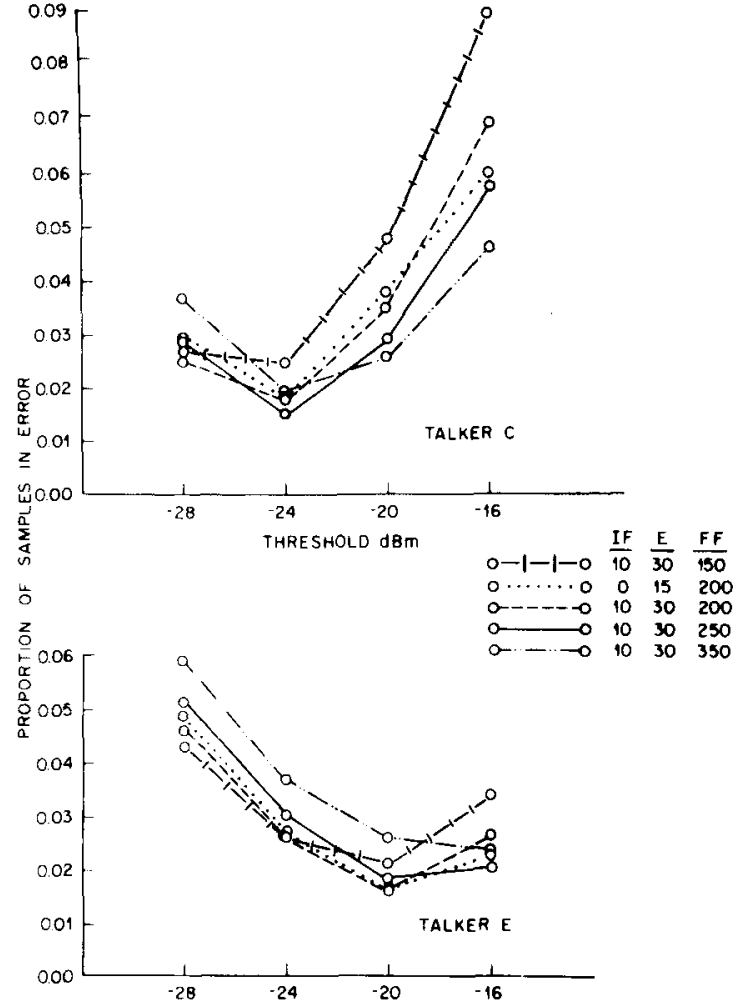

Figure 2. Proportion of samples in error as a function of system parameters for two talkers.
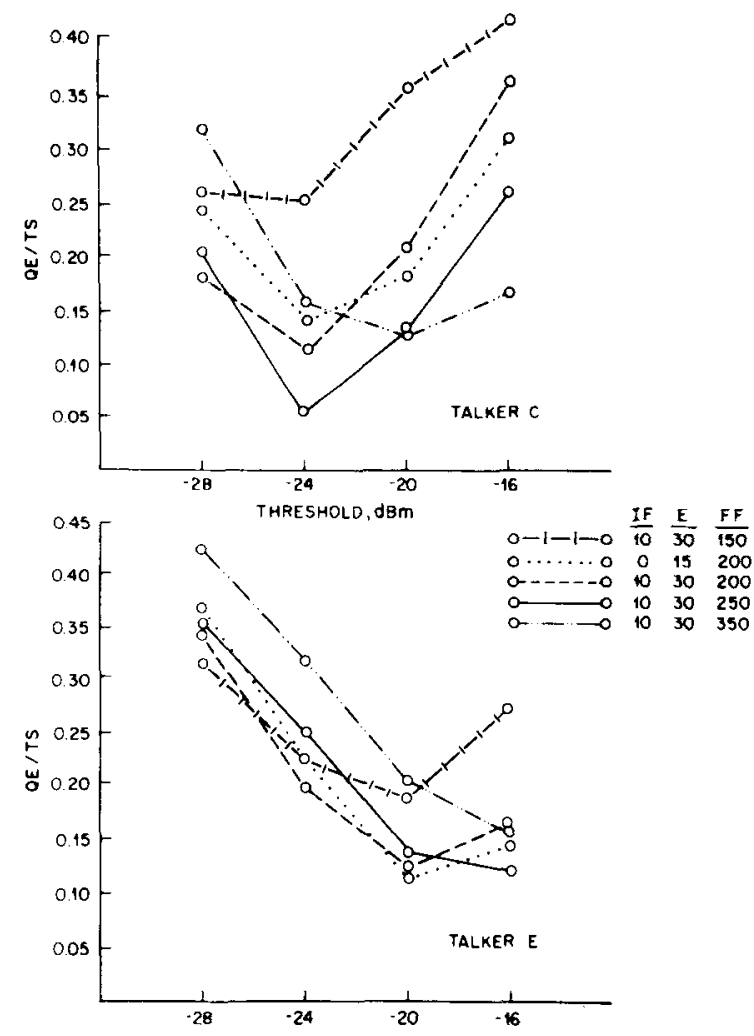

Figure 3. Proportion of qualitative error as a function of system parameters for two talkers. 


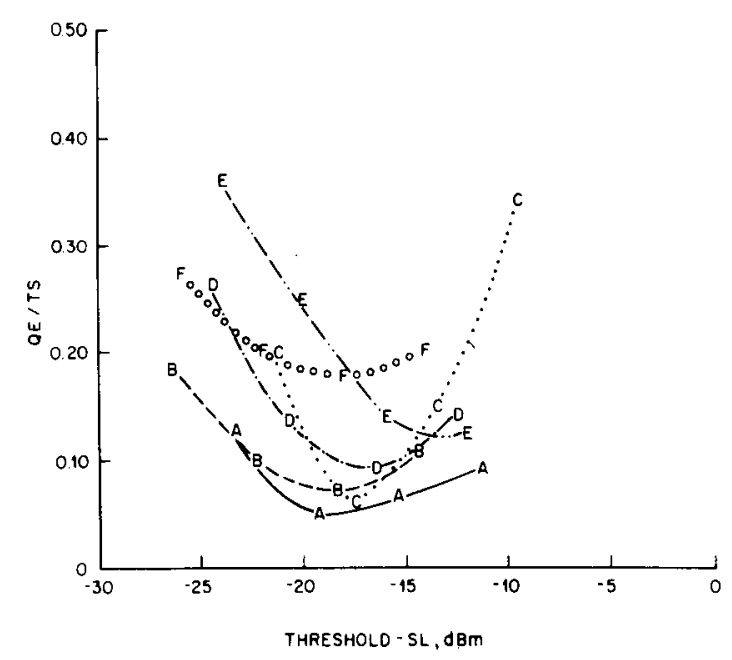

Figure 4. Proportion of qualitative error at optimum E-F parameter values as a function of threshold adjusted for speech level, for all six talkers.

illustrate another point: Namely, that neither the optimum threshold nor the way threshold and E-F parameters interact is independent of speaker.

A study of all six speakers reveals several generalizations. (1) The best performance at any threshold was delivered by the parameter set $\mathrm{IF}=10, \mathrm{E}=30, \mathrm{FF}=250$ in four cases; the other two speakers exhibited slight advantages for 0-15-200 (Speaker E, Figure 3) or 10-30. 200 (Speaker F, not shown). (2) Optimum threshold for optimum E-F values was either -20 or $-24 \mathrm{dBm}$ in all cases. (3) Errorless performance was achieved in no case. The absolute values of the error rate, as expressed by $\mathrm{QE} / \mathrm{TS}$, are in sharp contrast to those typical of sample-by-sample comparisons. The best performance under any parameter set ranged from $\mathrm{QE} / \mathrm{TS}=.04$ to .13 over the six talkers.

Threshold optimization. In an effort to clarify the qualitative effects of the threshold parameter and to simplify the optimization rule, the threshold $(\mathrm{T})$ was expressed relative to the speech level (SL) of each talker. The latter was measured by an objective technique (Bricker, 1965) that measures RMS voltage during speech spurts and is referenced to the $\mathrm{dBm}$ scale. In Figure 4, the QE/TS values for each talker under the generally optimal E-F parameter set (10-30-250) are plotted as a function of T-SL. Smooth curves are fitted by eye. All functions except that for Talker $\mathrm{E}$ pass through a minimum in the vicinity of $\mathrm{T}-\mathrm{SL}=-17$ to $-19 \mathrm{~dB}$. [It will be recalled that Talker $\mathrm{E}$ exhibited a minimum for E-F parameters of 0-15-200 (Figure 3).] Note that this minimum is much more sharply defined for some talkers (e.g., C) than for others (e.g., A). It appears that relating threshold to speech level is imperative, but that only fair-to-good performance at the qualitative level can be expected.

\section{Quantitative}

Because there is always some qualitative error, there is no straightforward way to assign the events (talkspurts and pauses) of one data file to those of another for purposes of comparing them on a purely quantitative (measurement accuracy) basis. We resorted, therefore, to examining the ECDFs for talkspurts and pauses. It must be borne in mind that discrepancies between criterion and automatic ECDFs may reflect qualitative errors as well as measurement error.

Table 1 shows mean talkspurt and pause durations as measured by the 10-30-250 parameter set at two thresholds, along with the criterion means and the standard errors of those means, for all six talkers. The two thresholds bracket the criterion means in half the cases, suggesting that setting threshold relative to speech level could optimize quantitative as well as qualitative performance. Furthermore, the better threshold approximates the criterion sample talkspurt mean to within a fraction of a standard error in all cases, while these means themselves cover a range, over talkers, of about seven times their average standard error. That is to say, automatic talkspurt measurement produced estimates of mean durations for the six talkers that were very similar to those obtained from the criterion method. Measurements of pauses are of less interest because these distributions include some very long listening pauses that profoundly affect the means and standard errors.

Table 1 and extensive examination of ECDFs for various parameter combinations support the following generalizations: (1) With threshold optimized, the dis-

Table 1

Mean Talkspurt and Pause Durations of Six Talkers for the 10-30-250 Parameter Set at Two Thresholds and for the Criterion Method

\begin{tabular}{|c|c|c|c|c|c|c|c|}
\hline & & Talker A & Talker B & Talker C & Talker D & Talker E & Talker $\mathrm{F}$ \\
\hline & \multicolumn{7}{|c|}{ Talkspurts } \\
\hline Criterion & $\begin{array}{l}\mathrm{M} \\
\sigma \mathrm{M}\end{array}$ & $\begin{array}{r}1.282 \\
.076\end{array}$ & $\begin{array}{r}1.396 \\
.071\end{array}$ & $\begin{array}{r}2.035 \\
.110\end{array}$ & $\begin{array}{r}1.417 \\
.093\end{array}$ & $\begin{array}{r}1.766 \\
.132\end{array}$ & $\begin{array}{r}1.685 \\
.113\end{array}$ \\
\hline \multirow[t]{2}{*}{ Automatic } & $\begin{array}{l}-20 \\
-24\end{array}$ & $\begin{array}{l}1.245 \\
1.308\end{array}$ & $\begin{array}{l}1.424 \\
1.504\end{array}$ & $\begin{array}{l}1.765 \\
2.025\end{array}$ & $\begin{array}{l}1.352 \\
1.469\end{array}$ & $\begin{array}{l}1.822 \\
1.872\end{array}$ & $\begin{array}{l}1.674 \\
1.900\end{array}$ \\
\hline & \multicolumn{7}{|c|}{ Pauses } \\
\hline Criterion & $\begin{array}{l}\mathbf{M} \\
\sigma \mathbf{M}\end{array}$ & $\begin{array}{r}2.155 \\
.176\end{array}$ & $\begin{array}{r}1.738 \\
.141\end{array}$ & $\begin{array}{r}2.040 \\
.195\end{array}$ & $\begin{array}{r}2.565 \\
.335\end{array}$ & $\begin{array}{r}2.227 \\
.231\end{array}$ & $\begin{array}{r}2.981 \\
.376\end{array}$ \\
\hline Automatic & $\begin{array}{l}-20 \\
-24\end{array}$ & $\begin{array}{l}2.230 \\
2.211\end{array}$ & $\begin{array}{l}1.819 \\
1.841 \\
\end{array}$ & $\begin{array}{l}1.935 \\
2.061 \\
\end{array}$ & $\begin{array}{l}2.558 \\
2.543 \\
\end{array}$ & $\begin{array}{l}2.250 \\
2.165 \\
\end{array}$ & $\begin{array}{l}3.034 \\
3.197 \\
\end{array}$ \\
\hline
\end{tabular}


tributions for the parameter set 10-30-250 closely approximate the location and general shape of criterion talkspurt and pause distributions; (2) final fill values greater or smaller than $200-250 \mathrm{msec}$ substantially misplace most ECDFs; (3) the number of brief talkspurts is overestimated by parameter sets that are otherwise acceptable. In view of the small proportion of samples in error attained by the better sets, it seems likely that the inflation of the talkspurt distribution at low duration values represents qualitative error (i.e., detection of noise bursts).

\section{DYADIC MEASUREMENTS}

To this point, we have discussed only the on-off pattern of each speaker without regard for the partner's pattern. When two partners' smoothed two-state data files are restored to their original temporal relationship, the dialogue is seen to be in one of four states at each sample point: $0=$ neither talking, $1=\mathrm{A}$ talking, $2=\mathrm{B}$ talking, $3=$ both talking. First- and second-order transitions among these states define a number of events that are the essence of the interactive speech activity pattern. For example, a run of twos, followed by a run of zeros and then another run of twos, signifies that Speaker B has paused during an utterance for a time equal to the length of the run of zeros. Similarly, a sequence of runs of the type $2-x-1$, where $x$ can mean State 0 , State 3 , or no intervening state, signifies that a switch from Speaker B to Speaker A has occurred.

Investigators have grouped these sequences in various ways and applied a variety of names to the groupings. In the absence of a generally accepted taxonomy, we define our classes and attach labels. In the section that follows on dyadic results, we shall refer to the following terms. Utterance: All the speech of one party, including pauses, that is preceded and followed by speech of the other party. Speaker switch: An interchange of speakerlistener roles. Encompassed talkspurt: A talkspurt of one party that is preceded, accompanied, and followed by uninterrupted speech from the other party. Withinutterance pause: A silence preceded and followed by speech of one party.

These events are illustrated in Figure 5, where onsets and terminations are labeled " $\mathrm{O}$ " and " $\mathrm{T}$," respectively. Note that the interval associated with a speaker switch can be positive (when silence separates one speaker's termination from the other's onset) or negative (when one's onset precedes the other's termination). An encompassed talkspurt is not considered an utterance, nor does it constitute a speaker switch. For purposes of computing utterance durations, ${ }^{1}$ talkspurts and pauses between speaker switches are summed.

The automatic measurement of dyadic phenomena was carried out under 12 conditions, generated by crossing the four threshold levels used for on-off pattern studies with three sets of smoothing parameters. These sets consisted of the two best IF-E-FF sets (10-30-200 and 10-30-250) and a simulated 300-msec sampling condition. In this condition, if any six consecutive original (unsmoothed) $5-\mathrm{msec}$ samples had the value "one" during a succession of 60 samples, the entire $300-\mathrm{msec}$ interval was set equal to one, otherwise equal to zero. This rule was applied for each successive 300-msec segment, with no running averaging, erasing, or filling. This condition was included because similar approaches appear in the literature (Cappella \& Streibel, 1979; Casotta et al., 1964).

For each of the 12 primary conditions and the criterion scoring, partners' two-state data files were aligned and processed by a program that generated a four-state time series for each dyad. Each four-state MS time series was then compared with the criterion, in a manner analogous to that used for two-state patterns, to generate a difference series. Again, qualitative and quantitative errors were sorted out. Within-utterance pauses were analyzed by a more limited method that compared the means and number of cases of the criterion scoring with one parameter set.

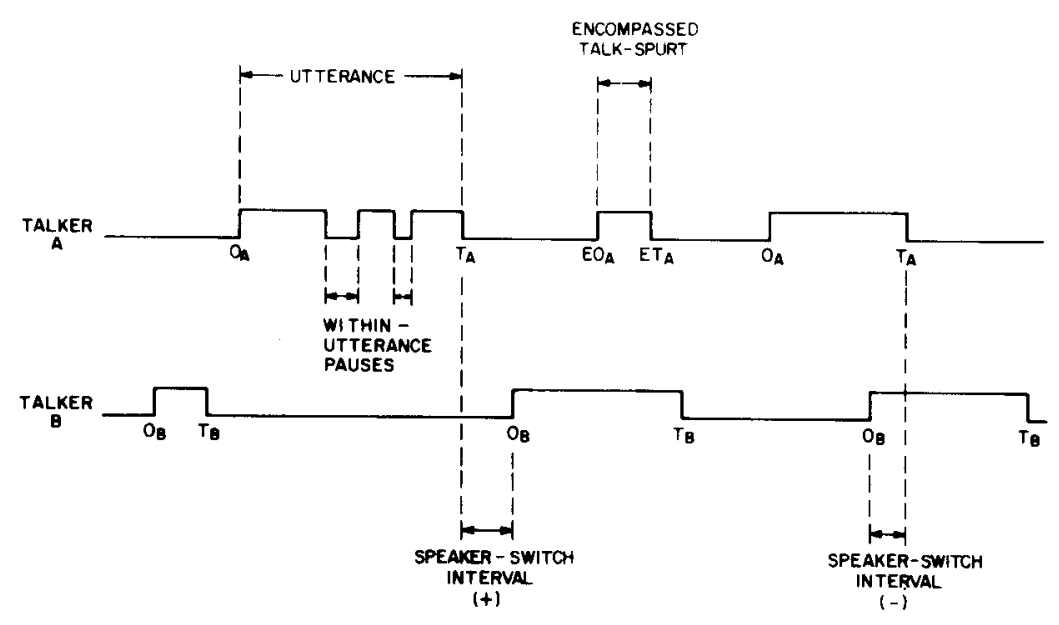

Figure 5. Schematic representation of dyadic events; $O=$ onset, $T=$ termination (of activity). 


\section{DYADIC RESULTS}

\section{Qualitative}

The criterion scoring identified 633 speaker switches and 162 encompassed talkspurts in the three conversations combined. Because the number of cases is small, most results are presented in terms of this total threeconversation sample.

The total number of differences (extra or missing speaker switches and encompassed talkspurts) is shown for each parameter set and threshold in Table 2. There is a clear superiority of the erase-and-fill conditions over the 300-msec sampling condition, and the best performance (about $4 \%$ of total events) was achieved at $-20 \mathrm{dBm}$ by 250 -msec final fill. Further results are for the 250 -msec condition only.

Table 3 displays extra and missing speaker switches and encompassed talkspurts for the 10-30-250 condition at four thresholds. For the speaker-switch class of events, performance was good ( $2 \%-3 \%$ error at $-20 \mathrm{dBm}$ or $-24 \mathrm{dBm}$ ) and relatively insensitive to threshold setting. The same cannot be said for encompassed talkspurts, however. Spurious and missing events total about $15 \%$ of base frequency at best, and lower threshold settings rapidly increase the number of false events.

The results on number of within-utterance pauses, for a threshold of $-24 \mathrm{dBm}$ and $250 \mathrm{msec}$ final fill, are summarized separately for each talker in Table 4 . The error rate is $16 \%$ overall, ranging from $4 \%$ to $40 \%$ over talkers. The fact that two-thirds of the errors were of the missing. pause type suggests that a higher threshold might have improved performance. Nevertheless, it is clear that performance on the subset of pauses that occur while a

Table 2

Number of Extra or Missing Speaker Switches and Encompassed Talkspurts for Three E-F Parameter Sets at Four Thresholds

\begin{tabular}{lrrrr}
\hline & \multicolumn{4}{c}{ Threshold (in dBm) } \\
\cline { 2 - 5 } & -28 & -24 & -20 & -16 \\
\hline Low Sampling Rate & 168 & 136 & 130 & 139 \\
200-Msec Fill & 138 & 54 & 51 & 64 \\
250-Msec Fill & 128 & 52 & 33 & 51 \\
\hline
\end{tabular}

Table 3

Extra and Missing Speaker Switches and Encompassed Talkspurts as Proportions of Criterion Count for Optimum E-F Parameters at Four Thresholds

\begin{tabular}{lcccc}
\hline & \multicolumn{4}{c}{ Threshold (in dBm) } \\
\cline { 2 - 5 } & -28 & -24 & -20 & -16 \\
\hline Extra & .016 & .013 & .019 & .032 \\
Missing & .028 & .016 & .005 & .009 \\
& & Speaker Switches \\
Extra & .689 & .227 & .091 & .068 \\
Missing & .076 & .053 & .061 & .121 \\
\hline
\end{tabular}

Table 4

Within-Utterance Pauses for Six Talkers: Criterion-Scored Number, Extra, and Missing Pauses at -24 dBm/10-30-250

\begin{tabular}{|c|c|c|c|c|}
\hline \multirow[b]{2}{*}{ Talker } & \multicolumn{3}{|c|}{ Number } & \multirow{2}{*}{$\begin{array}{c}\text { Proportion } \\
\text { of Error }\end{array}$} \\
\hline & Criterion & Extra & Missing & \\
\hline$A$ & 104 & 8 & 4 & .115 \\
\hline B & 177 & 6 & 20 & .147 \\
\hline C & 104 & 3 & 1 & .038 \\
\hline D & 98 & 2 & 8 & .102 \\
\hline E & 67 & 9 & 18 & .403 \\
\hline$F$ & 54 & 5 & 13 & .333 \\
\hline Total & 604 & 33 & 64 & .161 \\
\hline
\end{tabular}

speaker is holding the floor is worse than that for the entire set (cf. two-state results), which includes listening pauses.

\section{Quantitative}

The distributions of utterance durations, according to the criterion method and four thresholds for the 10-30. 250 parameter set, are shown in Figure 6. While the differences are in the expected direction, the spread is small. A threshold value of $-24 \mathrm{dBm}$ tracks the criterion distribution especially well, and no parameter set exhibits the excess of short values found for talkspurts.

Figure 7 shows the speaker-switching interval distributions on a linear abscissa, rather than on the logarithmic scale that has been used to this point. All distributions are smoothly sigmoid, although a substantial proportion of cases ( $25 \%$ for the criterion distribution) are negative. It therefore seems reasonable to define a class of events in this way. Again, the $-24 \mathrm{dBm}$ threshold gives a somewhat better approximation of the criterion distribution than do the others.

The encompassed talkspurt distributions are shown in Figure 8. None of the automatic methods gives a good account of this class of events, but some clues as to how to improve them are given by the data. Note the reversal of the order of thresholds below $100 \mathrm{msec}$. The lowest threshold produces the highest count, but above $300 \mathrm{msec}$, they have reverted to their.usual order for the active state. Another clue from the qualitative results (Table 3) is that lower thresholds find an excessive number of encompassed "talkspurts" not considered to be speech by the human observer. Together, these results suggest that a final erase of about $100 \mathrm{msec}$ would be of some benefit. Its cost, however, would be the loss of the approximately $6 \%$ of true events less than $100 \mathrm{msec}$ in duration. An analysis of these brief events indicates that the great majority were acoustic noises made by the listener's vocal/breathing apparatus or body or limb movements.

Criterion values of mean within-utterance pause durations, along with those measured by one parameter set, are shown in Table 5. While the quantitative error is generally considerably smaller than the qualitative, large (or small) values of the two types of error do not neces- 


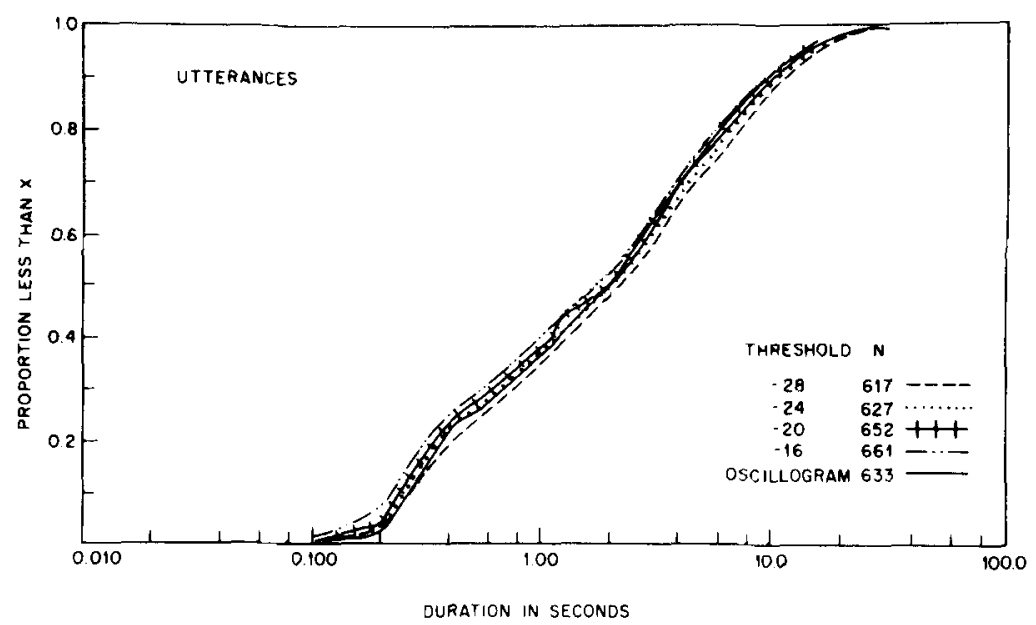

Figure 6 Distributions of utterance durations for the criterion scoring and the 10-30-250 parameter set at four thresholds.

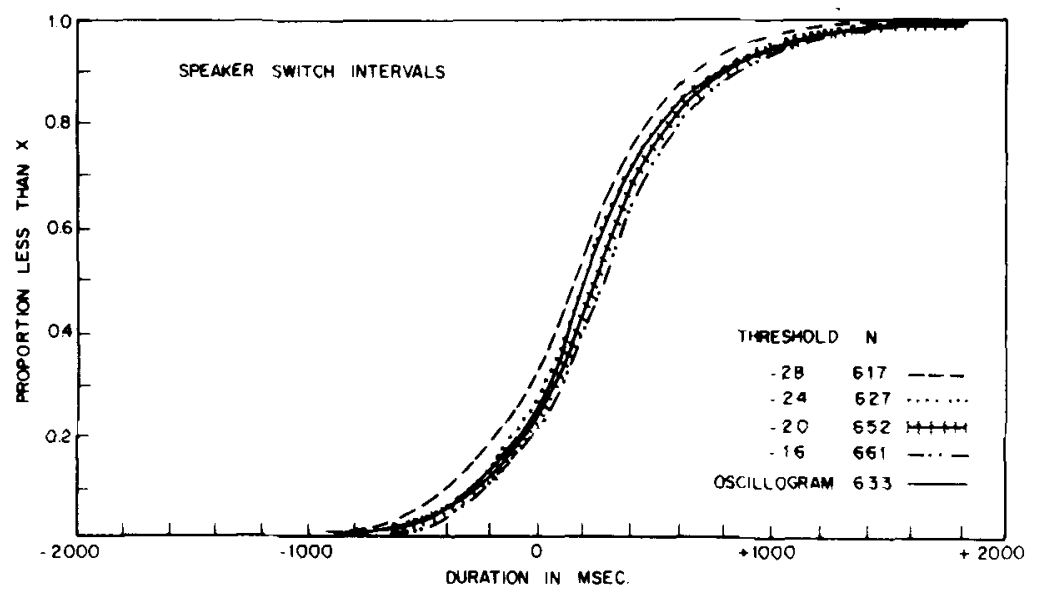

Figure 7. Distributions of speaker-switching intervals for the criterion scoring and the 10-30-250 parameter set at four thresholds.

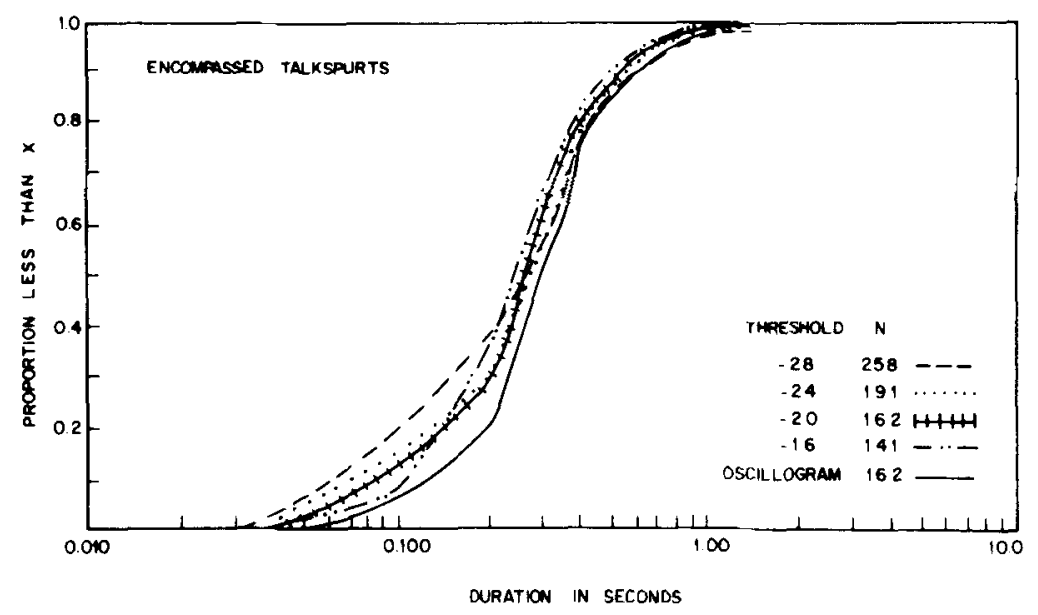

Figure 8. Distributions of encompassed talkspurt durations for the criterion scoring and the 10-30-250 parameter set at four thresholds. 
Table 5

Mean Within-Utterance Pause Durations (in Milliseconds) for the Criterion Scoring and the 10-30-250 Parameter Set at $-24 \mathrm{dBm}$

\begin{tabular}{cccc}
\hline Talker & Criterion & $10-30-250$ & Percent Error \\
\hline A & 614 & 623 & 1.5 \\
B & 599 & 641 & 7.0 \\
C & 619 & 578 & 6.6 \\
D & 510 & 490 & 3.9 \\
E & 711 & 724 & 1.8 \\
F & 595 & 625 & 5.0 \\
\hline
\end{tabular}

sarily occur for the same talkers (cf. Table 4). Thus, for Talker $\mathrm{E}$ the relatively accurate estimate of the mean must involve compensating errors, while the accurate identification of within-utterance pauses of Talker $\mathrm{C}$ is achieved at the cost of over $6 \%$ underestimation of mean duration.

\section{DISCUSSION}

The class of devices for measuring conversational speech activity patterns investigated here seems capable of giving a good account of utterances and speaker switches. With parameters optimized for these events, the same devices produce data of little practical value on pauses within utterances and encompassed talkspurts.

These strengths and weaknesses have implications for those who would use automated methods to investigate hypotheses about effects of personality, situational, or modality variables on conversational speech patterns. The relative robustness of identifying speaker switches implies that acoustic methods can be relied upon in investigations requiring the measurement of utterance durations and/or speaker-switching rates. Typical of questions involving interswitch intervals is that addressed by Matarazzo, Weitman, Saslow, and Wiens (1963), who studied the influence of interviewers' speech lengths on those of interviewees. One might also investigate in this manner whether overall switching rates are affected by visual access and whether utterance-length symmetry is related to role or dominance relations between members of a dyad.

Positive relationships between the switching pauses of interactors have been reported for interviewer-interviewee pairs (Matarazzo \& Wiens, 1967) and randomly paired conversers (Jaffe \& Feldstein, 1970). The present results (Figure 7) suggest that findings on questions of this sort, too, can be treated with confidence if generated from a properly adjusted automatic device.

The measurement of pauses within utterances has figured in a variety of investigations, but results have not been entirely clear and consistent. We suggest that some of the confusion may stem from the uncertainty of pause measurement as demonstrated here. The great dynamic range of syllable peaks exerts pressure to lower thresholds and increase fill-in times, while the prevalence of nonspeech noises during brief pauses exerts pressures in the opposite direction. In the present investigation, the best compromise between these pressures resulted in an error rate for pause detection that was about five times that for speaker switches. At the same time, the accuracy of measurement of the durations of withinutterance pauses was not as good as that attained for utterances and switching intervals.

A final class of behaviors of interest to social scientists concerns simultaneous speech by both members of a dyad. Meltzer, Morris, and Hayes (1971) provide a valu. able discussion of the concept of interruption and its relation to attempts to dominate. Jaffe and Feldstein (1970) found that mean simultaneous speech length decreased when visual access was denied, while Argyle, Lalljee, and Cook (1968) found that the number of interruptions per unit time increased under this condition. Brady (1968) presented distributions of three separate events related to interruptions, none of which is the same as the "encompassed talkspurt" event measured here. The latter would be classified by Meltzer et al. as an unsuccessful interruption attempt. The present study demonstrates that an automatic device set to do a good job on negative speaker-switching intervals (successful interruption attempts?) fails grossly on encompassed talkspurts, chiefly in that it detects too many brief events.

The present paper follows the tradition established by Norwine and Murphy (1938) of grouping positive and negative speaker-switching intervals together, whereas every other investigator has formed a single class for simultaneous speech, whether or not terminated by a speaker switch. Our reasons for this choice include the following:

1. Speaker switches dependably segment the interaction and can be easily identified and accurately located.

2. It is natural to examine the antecedent conditions, given that a speaker switch has occurred, if one wishes to describe interactional structure; the study of consequences, according to Duncan, Brunner, and Fiske (1979), has more to do with interactional strategy.

3. Our classification seems less judgmental than one which regards all instances of simultaneous speech as interruption attempts. Not all negative switching intervals are necessarily considered interruptions by the speaker who yields the floor, who might very well be trailing off with a sociocentric sequence (Duncan \& Fiske, 1977), having given other switching signals. Similarly, the present data show that not all encompassed talkspurts detected by an acoustic device represent speech, let along unsuccessful attempts to interrupt.

It is necessary for Jaffe and Feldstein (1970) to consider simultaneous speech as a single class in their four-state model, since they are interested in modeling the interaction process left to right. Further, Meltzer et al. (1971) have established the utility of this clas- 
sification by demonstrating a social-psychophysical relationship between speech levels and outcomes of simultaneous speech states. From the point of view of evaluating automatic acoustic speech detectors, however, the present grouping divides these events into one class on which the devices perform well and another on which they do poorly. The findings of Meltzer et al. may help to explain the difference. Successful interruptions (negative switching intervals) generally involve higher speech levels by the interrupter; these would be reliably detected whether or not accompanied by noise. Unsuccessful attempts and extraneous noises are both lower in level and brief, so that the class "encompassed talkspurts" as seen by an acoustic device contains a mixture of speech and noise events. All in all, it would seem that investigators truly interested in interruptions should not entrust their measurement to an automatic device.

We turn now to a consideration of the significance of the present results for the evaluation of existing conversational speech activity measurement techniques. It seems clear that a 300-msec sampling interval (Cappella \& Streibel, 1979; Jaffe \& Feldstein, 1970) provides insufficient resolution to make the distinctions perceived by our criterial scorer. Our 300-msec condition detected $20 \%$ too few speaker switches and produced larger mean durations of talkspurts and pauses as a consequence of missing brief events. This sampling interval also obscures a feature of both utterance and talkspurt distributions first reported by Norwine and Murphy (1938) and replicated here (Figure 6), namely, that they are bimodal. It is likely that the mode at about a quarter of a second is due largely to sounds emitted by the person who does not have the floor-what Duncan (1974) has called back channel signals, and Fries (1952) distinguished as brief signals of continued attention. It would seem ill-advised for investigators of dyadic interaction to overlook this distinction. Jaffe and Feldstein's (1970) finding of an exponential distribution for talkspurts, which Cappella and Streibel (1979) seem reassured to find in their own data, may simply be an artifact of the unduly coarse categorization imposed by the 300-msec interval.

As for directions in which automatic devices might be developed to further improve their match with human judgment, we feel the following avenues are promising:

1. Detectors might take advantage of some form of frequency or periodicity analysis of speech (Atal \& Rabiner, 1976). It might be desirable, for example, to reject an aperiodic noise burst even greater than $30 \mathrm{msec}$ long as nonspeech unless it is found temporally close to a periodic (voiced) sound.

2. The poor performance of automatic devices with regard to encompassed talkspurts (interruptions) might be improved by making a second pass through the data after establishing who has the floor. On the second pass, more stringent criteria, such as a $100-\mathrm{msec}$ minimum duration, might be applied to the talkspurts of the nonfloor holder.

3. The use of human judges in conjunction with mechanized devices should not be ruled out. Given a means of collecting synchronized multiple behavior time-lines (Bricker \& Wish, Note 4), human judges could make one or two real-time passes through recorded material pressing keys to identify bona-fide interruption attempts, filled pauses, and perhaps "autointerruptions" (sudden changes of direction by a single speaker following abandonment of a sentence before completion, often with little or no silent gap). Although such judges exhibit constant and variable error, software could assign their marks to nearby acoustic changes located precisely by machine on another pass.

Exploration of the extent to which automatic conversational speech activity scoring can be brought into correspondence with human judgment would seem to be in order. Until this correspondence has been maximized, however, it is important to recognize that machinescored "interruptions" and "pauses" do not correspond one to one with their perceptual counterparts.

\section{REFERENCE NOTES}

1. Höhne, H. D. Influence of long transmission delays and reverberation on the telephone conversations of test persons (Tech. Rep. 97). West Berlin: Heinrich-Hertz-Institut, 1968.

2. Phillips, D. A., Treurniet, W. C., Tigges, W. S., \& Lewis, P. The analysis of vocal interaction: $A$ report on methodology (Tech. Note 684). Ottawa: Department of Communications, Communications Research Center, March 1977.

3. Jensen, O. C. Speech detector. Unpublished manuscript, Bell Laboratories, October 1962.

4. Bricker, P. D., \& Wish, M. A new program of research on interpersonal communication: Laboratory facility and some early results. Paper presented at the National Telecommunications Conference, 1978.

\section{REFERENCES}

Argyle, M., Latl.jee, M., \& Cook, M. The effects of visibility on interaction in the dyad. Human Relations, 1968, 21, 3-17.

Atal., B. S., \& Rabiner, L. R. A pattern-recognition approach to voiced-unvoiced-silence classification with applications to speech recognition. IEEE Transactions on Acoustics, Speech, and Signal Processing, 1976, ASSP-24, 201-212.

Bai.ts, R. F. Interaction process analysis: A method for the study of small groups. Reading, Mass: Addison-Wesley, 1950.

Brady, P. T. A technique for investigating on-off patterns of speech. Bell System Technical Journal, 1965, 44, 1-22.

Brady, P. T. A statistical analysis of on-off patterns in 16 conversations. Bell System Technical Journal, 1968, 47, 73-91.

Bricker, P. D. Technique for objective measurement of speech levels. Journal of the Acoustical Society of America, 1965, 38, 361-362.

Cappel.l.A, J. N., \& Streibei, M. J. Computer analysis of talksilence sequences: The FIASSCO system. Behavior Research Methods \& Instrumentation, 1979, 11, 384-392.

Cassotta, L., Feldotein, S., \& Jaffe, J. AVta: A device for automatic vocal transaction analysis. Journal of the Experimental Analysis of Behavior, 1964, 7, 99-104.

Duncan, S. D., JR. On the structure of speaker-auditor interaction during speaking turns. Language in Society, 1974, 2, $161-180$. 
Duncan, S. D., Jr., Brunner, L. J., \& Fiske, D. W. Strategy signals in face-to-face interaction. Journal of Personality and Social Psychology, 1979, 37, 301-313.

Duncan, S. D., Jr., \& Fiske, D. W. Face-to-face interaction: Research, methods, and theory. Hillsdale, N.J: Erlbaum, 1977.

Fries, C. C. The structure of English. New York: Harcourt \& Brace, 1952.

Goldman-Eisler, F. The determinants of the rate of speech output and their mutual relations. Journal of Psychosomatic Research, 1956, 1, 137-143.

Jaffe, J., \& Feldstein, S. Rhythms of dialogue. New York: Academic Press, 1970.

Matarazzo, J. D., Weitman, M., Saslow, G., \& Wiens, A. N. Interviewer influence on durations of interviewee speech. Journal of Verbal Learning and Verbal Behavior, 1963, 1, 451-458.

Matarazzo, J. D., \& Wiens, A. N. Interviewer influence on duration of interviewee silence. Journal of Experimental Research in Personality, 1967, 2, 56-69.

Meltzer, L., Morris, W. N., \& Hayes, D. P. Interruption out- comes and vocal amplitude: Explorations in social psychophysics. Journal of Personality and Social Psychology, 1971, 18, 392-402.

Norwine, A. C., \& MurPhy, O. J. Characteristic time intervals in telephonic conversation. Bell System Technical Journal, 1938, 17, 281-291.

Ramsay, R. W., \& LAW, L. N. The measurement of duration of speech. Language and Speech, 1966, 9, 96-102.

WisH, M. Dimensions of dyadic communication. In S. Weitz (Ed.), Nonverbal communication (2nd ed.). New York: Oxford University Press, 1979.

\section{NOTE}

1. "Utterance duration" as used here is equivalent to "turn length" (Duncan \& Fiske, 1977) and "floor time" (Jaffe \& Feldstein, 1970).

(Received for publication October 2, 1980; accepted October 20,1980.) 